\title{
Relationship between vascular stiffness and stress myocardial perfusion imaging in asymptomatic patients with diabetes
}

\author{
Cornelis J. Roos • Roxana Djaberi • Joanne D. Schuijf • Eelco J. de Koning • \\ Ton J. Rabelink • Jan W. Smit • Alberto M. Pereira • Imad Al Younis • \\ Bernies van der Hiel • Arthur J. Scholte • Jeroen J. Bax • J. Wouter Jukema
}

Received: 15 February 2011 / Accepted: 22 July 2011 /Published online: 18 August 2011

(C) The Author(s) 2011. This article is published with open access at Springerlink.com

\begin{abstract}
Purpose Vascular stiffness may potentially be used as a screening tool to identify asymptomatic patients with diabetes with abnormal myocardial perfusion. The purpose of this study was therefore to determine the association between vascular stiffness, measured in term of pulse wave velocity (PWV) and augmentation index (AIx), and abnormal myocardial perfusion imaging (MPI) in asymptomatic patients with diabetes.
\end{abstract}

Dr. Roos and Dr. Djaberi contributed equally to this work as first authors.

C. J. Roos $\cdot$ R. Djaberi $\cdot$ J. D. Schuijf $\cdot$ A. J. Scholte $\cdot$ J. J. Bax $\cdot$

J. W. Jukema $(\bowtie)$

Department of Cardiology, C5-P,

Leiden University Medical Center,

Postbus 9600, 2300 RC, Leiden, The Netherlands

e-mail: j.w.jukema@lumc.nl

\section{J. Roos $\cdot$ J. W. Jukema}

Interuniversity Cardiology Institute of the Netherlands (ICIN),

Utrecht, The Netherlands

E. J. de Koning • T. J. Rabelink

Department of Nephrology, Leiden University Medical Center, Leiden, The Netherlands

J. W. Smit $\cdot$ A. M. Pereira

Department of Endocrinology, Leiden University Medical Center,

Leiden, The Netherlands

I. Al Younis $\cdot$ B. van der Hiel

Department of Nuclear Medicine,

Leiden University Medical Center,

Leiden, The Netherlands

J. W. Jukema

Einthoven Laboratory of Experimental Vascular Medicine,

Leiden, The Netherlands
Methods Prospectively, 160 asymptomatic patients with diabetes (mean age 51 years, 87 men) underwent MPI with adenosine stress. The summed stress score (SSS) was determined in each patient according to a 17 -segment and five-point score. Abnormal MPI (SSS $\geq 3$ ) was classified as moderate (SSS 3-7) or severe (SSS $\geq 8$ ) MPI defects. Using applanation tonometry, the carotid-femoral PWV and the radial AIx corrected to 75 beats per minute were determined noninvasively.

Results MPI was abnormal in 61 patients (38\%), with severe MPI defects in 22 patients (14\%). Mean PWV increased with deteriorating MPI from $8.4 \pm 2.2 \mathrm{~m} / \mathrm{s}$ in normal MPI to $9.0 \pm$ $2.2 \mathrm{~m} / \mathrm{s}$ in moderate MPI defects $(p=0.11)$ and to $11.1 \pm 2.5 \mathrm{~m} / \mathrm{s}$ in severe MPI defects $(p<0.01)$. Likewise, mean AIx increased from $18.4 \pm 13.4 \%$ to $19.4 \pm 10.7 \%(p=0.66)$ and to $25.4 \pm 9.0 \%(p=0.03)$. After adjustment for age and other risk factors, PWV remained a significant predictor of severe MPI defects ( $p=0.01$, OR $1.50,95 \%$ CI $1.11-2.00$ ), whereas AIx was no longer significant $(p=0.20)$.

Conclusion Vascular stiffness measured by PWV is associated with severe MPI defects in asymptomatic patients with diabetes.

Keywords Diabetes mellitus · Vascular stiffness ·

Myocardial perfusion imaging $\cdot$ Coronary artery disease

\section{Introduction}

It is considered that the global prevalence of diabetes will approximately double in the next two decades [1]. Diabetes is associated with a marked increase in the incidence of cardiovascular morbidity and mortality, mainly attributable to coronary artery disease (CAD). Moreover, the presence 
and progression of CAD in diabetic patients is often asymptomatic, leading to more extensive disease at the time of diagnosis [2]. Since a delayed diagnosis of CAD considerably worsens the prognosis, early recognition of CAD could lead to more effectively targeted intervention and reduce morbidity and mortality in this population. Myocardial perfusion imaging (MPI) with SPECT is most commonly used to identify patients with CAD, and can accurately identify patients at increased cardiovascular risk [2-4]. However, based on recent data, a wide ranging routine MPI screening strategy of all asymptomatic patients with diabetes would appear to be ineffective [5]. Accordingly, a selective "prescreening" strategy using an initial test for the identification of patients with a higher likelihood of abnormal MPI followed by referral of only these patients to MPI may be preferred. Noninvasive assessment of vascular stiffness could represent a promising tool for this purpose. In several studies, a relationship between vascular stiffness and cardiovascular disease has been observed [6-8]. Assessment of vascular stiffness by means of pulse wave velocity (PWV) or pulse wave analysis for augmentation index (AIx) may therefore have the potential to serve as a marker of abnormal MPI. Although PWV and AIx have been extensively studied in the general population [9-13], fewer data are available concerning their relationship with CAD in asymptomatic patients with diabetes.

The aim of the current study was to prospectively assess the relationship between the noninvasive measures of vascular stiffness (PWV and AIx) with the presence and extent of myocardial perfusion defects as assessed by SPECT MPI in asymptomatic patients with diabetes.

\section{Methods}

Study population

Prospectively, 160 consecutive asymptomatic patients with diabetes were recruited from a routine outpatient clinic. Patients were referred to the cardiology outpatient clinic for risk assessment and cardiovascular screening. Anginal symptoms were ruled out using a self-completed questionnaire for encountered chest pain [14]. The American Diabetes association (ADA) criteria were used to identify diabetes and for further stratification into type 1 or 2 diabetes [15]. Patients were considered as having type 1 diabetes if laboratory analysis demonstrated autoantibodies to islet cells, insulin and glutamic acid decarboxylase or low levels of plasma c-peptide. Otherwise, patients were considered to have type 2 diabetes. Medical history and demographics were obtained. All patients underwent physical examination, and blood and urine laboratory testing. MPI was performed as part of the clinical work-up to determine the presence and extent of myocardial perfusion defects. Additional measurements of PWV and AIx were used to assess vascular stiffness.

\section{Cardiovascular risk factors}

Cardiovascular risk factors were defined according to the following criteria: positive family history for CAD (presence of CAD in first-degree family members, male $<55$ years and/or female $<65$ years), smoking (current smoking or smoking in the last 2 years), hypertension (blood pressure $>140 / 90 \mathrm{mmHg}$ or treatment with antihypertensive medication), body mass index, hypercholesterolaemia (total cholesterol level $>5.0 \mathrm{mmol} / \mathrm{l}$ or use of cholesterol-lowering medication), and microalbuminuria (urine albumin/creatinine ratio $\geq 3.5 \mathrm{mg} / \mathrm{mmol}$ ) [2]. Plasma haemoglobin A1c (HbA1c) was determined as a measure of glycaemic control.

\section{SPECT myocardial perfusion imaging}

\section{SPECT data acquisition}

ECG-gated adenosine ${ }^{99 \mathrm{~m}} \mathrm{Tc}$ sestamibi $\left({ }^{99 \mathrm{~m}} \mathrm{Tc} \mathrm{MIBI}\right)$ SPECT MPI was performed using a 2-day protocol, comprising stress imaging on the first day and a rest scan on the second day [16]. Antihypertensive treatment with beta-adrenergic blocking agents or calcium antagonists was stopped and patients were instructed to abstain from caffeine-containing products $24 \mathrm{~h}$ prior to the stress test. Vasodilator stress was induced by intravenous infusion of adenosine $140 \mu \mathrm{g} / \mathrm{kg}$ per minute for $6 \mathrm{~min}$, with a simultaneous hand-grip exercise. ${ }^{99 \mathrm{~m}} \mathrm{Tc}$ MIBI $(500 \mathrm{MBq})$ was injected intravenously after the third minute. Blood pressure and a 12-lead ECG were recorded throughout the adenosine infusion.

Images were acquired $2 \mathrm{~h}$ after injection of the radiopharmaceutical using a triple-head SPECT gamma camera (GCA 9300/HG; Toshiba, Tokyo, Japan) with lowenergy, high-resolution collimators. Images were acquired using a circular $360^{\circ}$ orbit, 60 projections and $40 \mathrm{~s}$ per projection, in compliance with the American Society of Nuclear Cardiology (ASNC) imaging guidelines. Images were processed to obtain the short-axis, vertical long-axis and horizontal long-axis sections, as well as polar map formats, normalized to maximal myocardial activity [16]. Patient motion was reviewed by examining the raw cine images. No attenuation or scatter correction was used.

\section{SPECT data analysis}

For semiquantitative visual interpretation, the myocardium was divided into 17 segments according to ASNC guidelines [16]. Tracer uptake in each segment was evaluated in 
consensus by two expert observers blinded to the patient's clinical characteristics and test results using a five-point scoring system ranging from 0 (normal uptake) to 4 (absent uptake). The summed stress score (SSS) was determined as the sum of the 17 segmental scores of the stress images. MPI was considered normal for SSS $<3$. In case of abnormal MPI, SSS in the range 3-7 were considered to represent moderate MPI defects, and SSS $\geq 8$ to represent severe MPI defects [17]. Finally, regional wall motion on gated SPECT images was evaluated to allow differentiation between true MPI abnormalities and diaphragmatic or breast attenuation artifacts.

\section{Assessment of vascular stiffness}

Measurements were derived and analysed noninvasively by applanation tonometry using a SphygmoCor system (Atcor Medical, Sydney, Australia). All measurements were performed in the morning in a quiet, temperature-controlled clinical research laboratory by a specially trained technologist blinded to the patient's clinical characteristics and test results. The patients were instructed to omit their morning medication and continue fasting until after the test. Assessment of PWV and pulse wave analysis commenced following a 10-min rest in a supine position after a state of constant heart rate and blood pressure had been reached.

\section{Pulse wave velocity}

The pulse waves were recorded at the common carotid artery and the femoral artery by sequential tonometry with simultaneous electrocardiographic gating. Pulse transit time was determined as the average of ten consecutive beats. The distance between the two sites was measured. Aortic PWV $(\mathrm{m} / \mathrm{s})$ was defined as the distance between the two recording sites travelled by the pulse wave divided by the transit time. Using system software, aortic PWV was determined semiautomatically. The validation and reproducibility of this semiautomatic method have been previously published [18]. Measurements were performed three times in each patient and averaged to obtain the mean aortic PWV.

\section{Pulse wave analysis}

The peripheral pressure waveforms were recorded from the radial artery at the wrist with a hand-held high-fidelity tonometer (Millar Instruments, Houston, TX) and calibrated by peripheral blood pressures at the brachial artery [7, 19]. The corresponding central aortic pressure waveform was generated by a validated generalized transfer function. The central aortic pressure waveform was analysed to identify the first shoulder of the pressure wave representing the incident wave attributable to left ventricular ejection. The merging point of the incident and the reflected wave (the inflection point) was then identified on the generated aortic pressure waveform. The absolute augmented pressure was the maximum systolic pressure minus the pressure at the inflection point. Subsequently, the AIx was defined as the absolute augmented pressure divided by the pulse pressure and expressed as a percentage [7, 8]. Finally, the AIx was normalized to a heart rate of 75 bpm (AIx@75). In each patient, three consecutive waveform recordings were averaged to obtain the mean AIx@75, which was used for statistical analysis.

Statistical analysis

Continuous variables are expressed as means \pm standard deviation and categorical variables as numbers (percentages). Firstly, associations of PWV and AIx@75 with baseline clinical risk factors were assessed using Pearson's correlation coefficient $(r)$ or Spearman's rank correlation coefficient $\left(r_{\mathrm{s}}\right)$ in case of dichotomous variables. Secondly, differences in the mean PWV and AIx@75 for each group of MPI results were evaluated with the independent $t$-test. Thereafter, with univariate logistic regression analysis potential predictors of severe MPI defects were identified. Subsequently, all potential predictors were analysed in a multivariate logistic regression model to identify the independent predictors of severe MPI defects. Additionally, patients were categorized according to PWV quartiles and for each quartile the prevalence of severe MPI defects was obtained. Subsequently, a global chi-squared analysis was used to determine the incremental predictive value of PWV over baseline characteristics. Thereafter, using receiver operating characteristic (ROC) curve analysis two cut-off values were chosen for $\mathrm{PWV}$, one for the detection of severe MPI defects with optimal sensitivity and specificity and the other for the exclusion of severe MPI defects with optimal sensitivity and negative predictive value. All statistical analyses were performed using SPSS software (version 16.0; SPSS, Chicago, IL). All $p$-values $<0.05$ were considered statistically significant.

\section{Results}

Patient characteristics

The study population comprised 160 asymptomatic diabetic patients. Their baseline characteristics are provided in Table 1.

SPECT myocardial perfusion imaging

The overall mean SSS was 3.1 \pm 4.1 (range 0-21). Abnormal MPI (SSS $\geq 3$ ) was observed in 60 patients (38\%), including 
Table 1 Baseline characteristics of the study population of 160 diabetic patients

\begin{tabular}{lc}
\hline Clinical factors & Mean \pm SD or number (\%) \\
\hline Age (years) & $51 \pm 12$ \\
Men & $87(54)$ \\
Type 2 diabetes & $91(57)$ \\
Diabetes duration (years) & $15 \pm 13$ \\
Insulin use & $125(78)$ \\
Family history of CAD & $75(47)$ \\
Smoking & $42(26)$ \\
Body mass index (kg/m $\left.{ }^{2}\right)$ & $28 \pm 6$ \\
Plasma haemoglobin A1c (mmol/l) & $8.2 \pm 1.7$ \\
Hypertension & $92(58)$ \\
Use of antihypertensive medication & $76(48)$ \\
ACE-inhibitor use & $43(27)$ \\
Beta-blocker use & $19(12)$ \\
Systolic blood pressure (mmHg) & $133 \pm 16$ \\
Diastolic blood pressure (mmHg) & $80 \pm 9$ \\
Hypercholesterolaemia & $107(67)$ \\
Cholesterol-lowering medication & $73(46)$ \\
Total cholesterol (mmol/l) & $4.8 \pm 1.1$ \\
Microalbuminuria & $39(24)$ \\
Aspirin use & $31(19)$ \\
\hline
\end{tabular}

moderate MPI defects (SSS 3-7) in 38 patients (24\%) and severe MPI defects ( $\mathrm{SSS} \geq 8$ ) in 22 patients $(14 \%)$.

Vascular stiffness

\section{Pulse wave velocity}

The overall mean PWV was $8.9 \pm 2.4 \mathrm{~m} / \mathrm{s}$. PWV was associated with age $(r=0.62, p<0.01)$, type 2 diabetes $\left(r_{\mathrm{s}}=\right.$ $0.23, p<0.01)$, diabetes duration $(r=0.30, p<0.01)$, body mass index $(r=0.22, p<0.01)$, hypertension $\left(r_{\mathrm{s}}=0.43, p<\right.$ $0.01)$ and microalbuminuria $(r=0.29, p<0.01)$. As shown in Fig. 1a, mean PWV was only slightly lower in patients with normal MPI than in patients with moderate MPI defects $(8.4 \pm 2.2 \mathrm{~m} / \mathrm{s}$ and $9.0 \pm 2.2 \mathrm{~m} / \mathrm{s}$, respectively; $p=0.11)$. However, the mean PWV was significantly higher in patients with severe MPI defects $(11.1 \pm 2.5 \mathrm{~m} / \mathrm{s} ; p<0.01)$.

\section{Association between pulse wave analysis and myocardial perfusion}

The mean AIx was $21.1 \pm 12.3 \%$ in the total population. Normalization for a heart rate of 75 beats per minute resulted in an overall mean AIx@75 of 19.6 $12.4 \%$. A significant association was observed between AIx@75 and the following risk factors: age $(r=0.47, p<0.01)$, male gender $\left(r_{\mathrm{s}}=-0.43, p<0.01\right)$, type 2 diabetes $\left(r_{\mathrm{s}}=0.30\right.$, $p<0.01)$, hypercholesterolaemia $\left(r_{\mathrm{s}}=0.17, p<0.03\right)$ and microalbuminuria $(r=0.26, p<0.01)$.

After stratification of the mean AIx@75 values according to SPECT MPI results, a trend similar to that for PWV was observed. Likewise, mean AIx@75 was slightly higher in patients with moderate MPI defects than in those with normal MPI (19.4 $\pm 10.7 \%$ and $18.4 \pm 13.4 \%$, respectively; $p=0.66)$, and was significantly higher in patients with severe MPI defects $(25.4 \pm 9.0 \%$; $p=0.03$; Fig. 1b).

\section{Predictors of severe myocardial perfusion defects}

As illustrated in Table 2, age, gender, smoking, HbA1c, microalbuminuria and both PWV and AIx@75 were identified as potential predictors of severe MPI defects in a univariate logistic regression model. Of note, after adjustment for age, gender, smoking, $\mathrm{HbA} 1 \mathrm{c}$ and microalbuminuria, the PWV remained a significant predictor of severe MPI defects $(p=0.01)$, whereas the AIx@75 was no longer significant.

As demonstrated in Fig. 2, the prevalence of severe MPI defects gradually increased with increasing PWV quartile. Importantly, in none of the patients in the lowest PWV
Fig. 1 Relationship between parameters of vascular stiffness and the extent of MPI defects as assessed by SPECT MPI. a Mean aortic PWV was higher in patients with abnormal MPI. The highest PWV was observed in patients with severe MPI defects. b The relationship between mean AIx@75 and MPI shows a similar trend
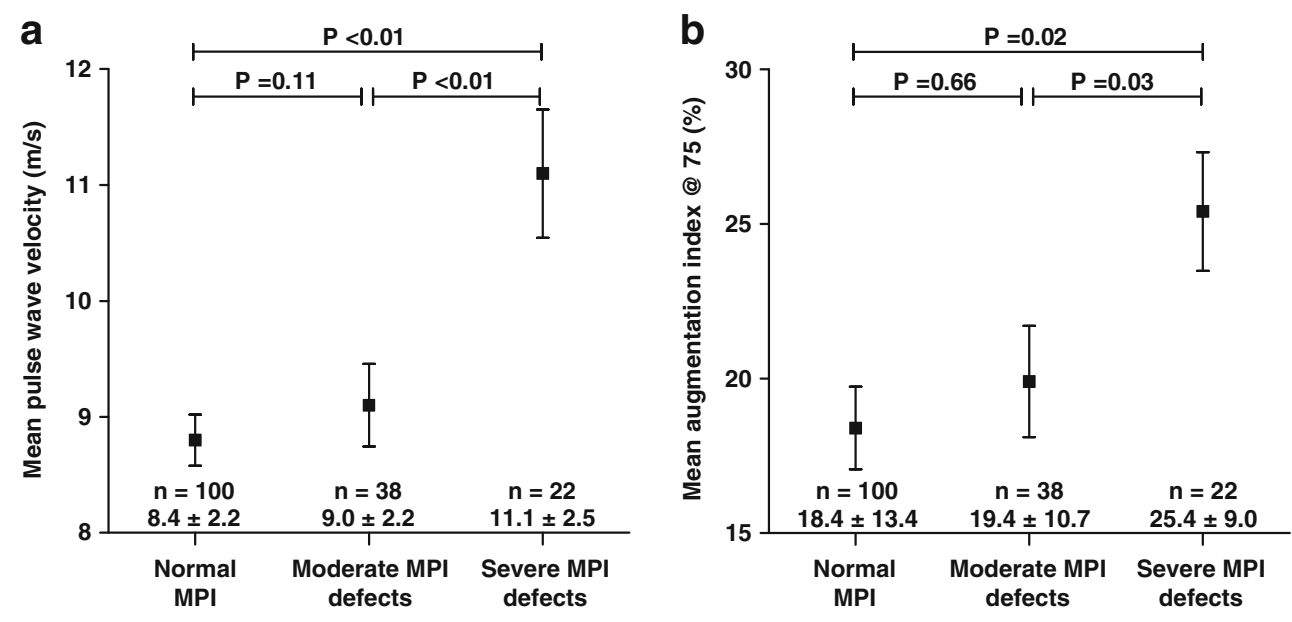
Table 2 Predictors of severe MPI defects (SSS $\geq 8$ ) on SPECT

\begin{tabular}{lcccc}
\hline Clinical characteristic & Exp $\beta(95 \% \mathrm{CI})$ & $p$-value & $\operatorname{Exp} \beta(95 \% \mathrm{CI})$ & $p$-value \\
\hline Age & $1.09(1.04-1.14)$ & $<0.01$ & $1.06(0.98-1.14)$ & 0.16 \\
Male gender & $3.30(1.15-9.45)$ & 0.03 & $6.35(1.47-27.41)$ & 0.01 \\
Type 2 diabetes & $1.47(0.55-3.90)$ & 0.44 & & \\
Diabetes duration (years) & $1.02(0.99-1.06)$ & 0.15 & & \\
Family history of CAD & $1.16(0.47-2.85)$ & 0.75 & & \\
Smoking & $3.80(1.48-9.77)$ & 0.01 & $5.74(1.35-24.46)$ & 0.02 \\
Body mass index (kg/m²) & $0.99(0.91-1.07)$ & 0.77 & & \\
HbA1c (mmol/l) & $1.28(1.00-1.65)$ & 0.05 & $1.52(1.03-2.25)$ & 0.03 \\
Hypertension & $1.98(0.73-5.41)$ & 0.18 & & \\
Hypercholesterolaemia & $1.65(0.57-4.79)$ & 0.36 & & \\
Microalbuminuria & $3.86(1.52-9.81)$ & 0.01 & $1.05(0.26-4.27)$ & 0.95 \\
PWV & $1.49(1.22-1.81)$ & $<0.01$ & $1.49(1.11-2.00)$ & 0.01 \\
AIx@75 & $1.06(1.01-1.11)$ & 0.02 & $1.05(0.97-1.14)$ & 0.20 \\
\hline
\end{tabular}

quartile were severe MPI defects present. Also, only a relatively small proportion of patients $(5 \%)$ in the second PWV quartile had severe MPI defects. In contrast, the prevalence of severe MPI defects was $20 \%$ in the third PWV quartile, and was $30 \%$ in the fourth quartile. Moreover, the addition of PWV to a model with baseline clinical risk factors age, gender and smoking for the prediction of severe MPI defects showed a significantly improved predictive value of PWV (Fig. 3).

ROC curve analysis for the detection of severe MPI defects showed the highest sensitivity and specificity (77\% and $75 \%$, respectively) with a PWV cut-off value of $9.8 \mathrm{~m} / \mathrm{s}$. An optimal sensitivity of $91 \%$ with an associated negative predictive value of $98 \%$ for the exclusion of severe MPI defects was found using a cut-off value of $9.2 \mathrm{~m} / \mathrm{s}$ for PWV (Fig. 4).

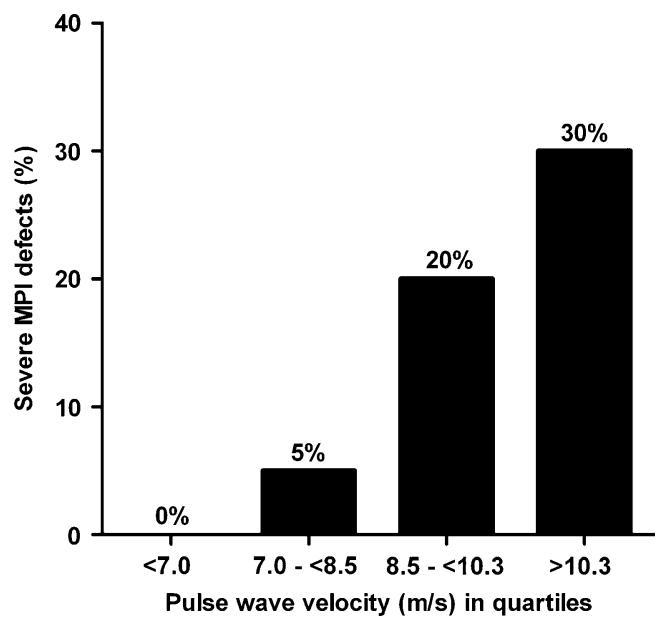

Fig. 2 Prevalence of patients with severe MPI defects per PWV quartile. The prevalence of severe MPI defects increased with increasing PWV. Of note, the prevalence of severe MPI defects chiefly increased in the third and fourth PWV quartile

\section{Discussion}

In the present study of asymptomatic patients with diabetes, vascular stiffness as assessed by PWV and AIx was greater in the presence of severe MPI defects. PWV was independently associated with severe MPI defects, whereas AIx was no longer significant after correction for other cardiovascular risk factors and PWV. Addition of PWV to a model of baseline clinical risk factors showed significant incremental value for the prediction of severe MPI defects. Furthermore, ROC curve analysis revealed a moderate to good sensitivity of $77 \%$ and a specificity of $75 \%$ for the detection of severe MPI defects, with a PWV cut-off value of $9.8 \mathrm{~m} / \mathrm{s}$. Conversely, changing the cut-off value to $9.2 \mathrm{~m} / \mathrm{s}$ resulted in a high sensitivity of $91 \%$ and negative predictive value of $98 \%$ for the exclusion of severe MPI defects. Accordingly, the current results indicate that noninvasive evaluation of vascular stiffness may be a practical tool for prescreening asymptomatic patients with

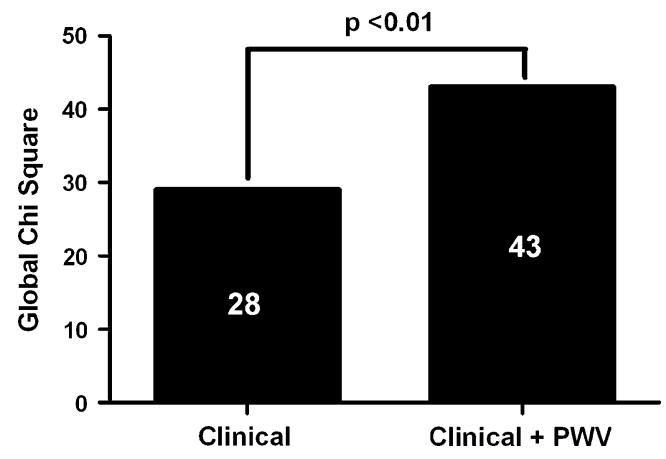

Fig. 3 Incremental predictive value of PWV for the detection severe MPI defects as shown by an increase in the value of global chisquared. Addition of PWV to a model with baseline clinical risk factors age, gender and smoking provided a significantly improved predictive value 
Fig. 4 Detection of severe MPI defects on SPECT by PWV. a ROC curve analysis for the detection of severe MPI defects yielded an optimal sensitivity and specificity of $77 \%$ and $75 \%$, respectively, with a PWV cut-off value of $9.8 \mathrm{~m} / \mathrm{s}$. b In contrast, optimization for the exclusion of severe MPI defects resulted in a cut-off value of $9.2 \mathrm{~m} / \mathrm{s}$ with a sensitivity of $91 \%$ and a corresponding negative predictive value of $98 \%$

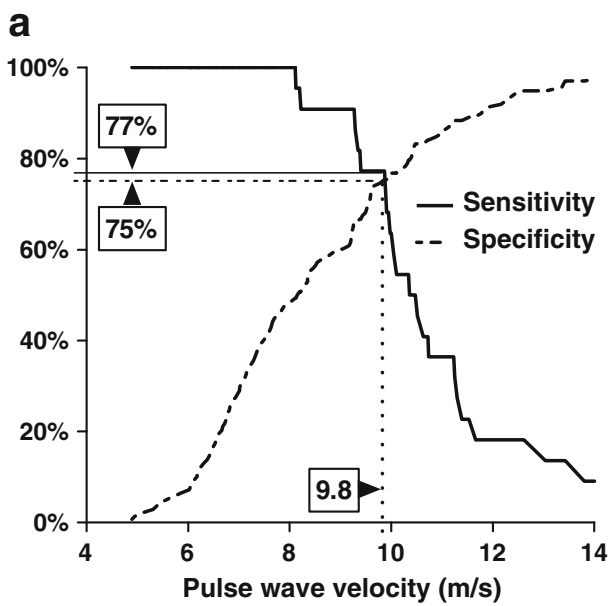

b

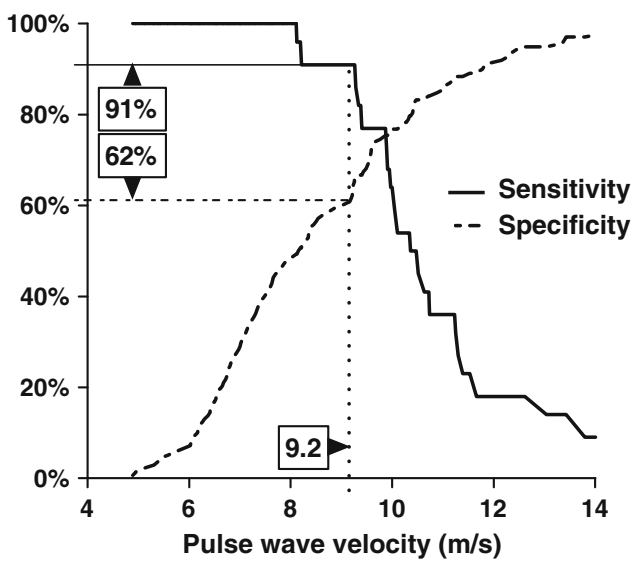

diabetes for differentiation into a higher and lower likelihood of abnormal MPI.

Vascular stiffness and relationship to CAD

In the general population the relationship between vascular stiffness and the presence of CAD has been confirmed in a considerable number of studies. Vascular stiffness measured as PWV or AIx not only is directly associated with the presence and severity of CAD on invasive coronary imaging [10], but also has incremental prognostic value for predicting cardiovascular events $[9,11-13]$. A recent meta-analysis $(15,877$ subjects, 17 studies, average followup of 7.7 years) has shown that the risk of cardiovascular events is increased twofold in patients with increased PWV [20]. Moreover, the predictive ability of PWV was shown to be even higher in patients with elevated baseline cardiovascular risk, supporting a role for PWV in high-risk populations such as patients with diabetes.

A few studies have specifically evaluated vascular stiffness in patients with diabetes. Cruickshank et al. evaluated the prognostic value of PWV for all-cause and cardiovascular mortality in 397 patients with diabetes with or without CAD. During a mean follow-up of 10.7 years, aortic PWV was an independent predictor for all-cause and cardiovascular mortality [21]. Additionally, Hatsuda et al. found in 595 patients with diabetes that PWV was significantly increased in 70 patients with established CAD [22]. Finally, Fukui et al. investigated 208 consecutive patients with type 2 diabetes and reported that AIx was significantly higher in 47 patients with previously confirmed CAD [23]. These observations indicate that markers of vascular stiffness may indeed be associated with CAD in patients with diabetes.

However, to our knowledge this is the first study in which PWV and AIx have been applied in asymptomatic patients with diabetes to prospectively identify the presence of CAD defined by the presence of (severe) MPI defects.
Although both PWV and AIx were higher in patients with severe MPI defects, only PWV was shown to be an independent predictor of severe MPI defects. These observations are in agreement with the previous literature as more discrepant results have also been reported in the general population using AIx as compared to PWV [24, 25].

Possibly, the more variable results with AIx may be explained by underlying methodological differences. Carotidfemoral PWV is a direct measure of vascular stiffness as determined by the intrinsic stress/strain relationship of the arterial wall and mean arterial pressure. Therefore PWV is considered as the 'gold-standard' [6-8]. In contrast, AIx is an indirect measurement derived from peripherally recorded pressure waveforms. Using a generalized transfer function the corresponding central arterial waveform is generated, from which AIx is determined. Therefore, AIx is influenced by multiple factors such as PWV, heart rate, diastolic blood pressure, peripheral circulation and endothelial function $[7,8]$. Furthermore, its discriminatory value may be less in the elderly [24, 25], and the use of the generalized transfer function may also be inappropriate in certain populations [2427]. In fact, Hope et al. recently evaluated the validity of this method in patients with diabetes and found that estimation of central pressures was prone to substantially greater error in this population [26]. Similar differences in accuracy have also been reported in relation to gender, indicating that AIx might be a less representative marker of vascular stiffness than PWV $[24,25]$. Conceivably, the weaker association between AIx and CAD as compared to PWV may therefore be explained by the fact that our study was performed in patients with diabetes while also including a high percentage of female patients.

\section{Clinical implications and perspectives}

At present, screening of asymptomatic patients with diabetes for CAD remains controversial. The majority of available data are based on CAD detection using SPECT 
MPI [3, 4]. In the present study, the prevalence of abnormal MPI was $38 \%$. In contrast, the recent DIAD trial demonstrated a much lower rate of abnormal MPI with only a few patients having severe MPI defects $[5,28]$. To a large extent, this discrepancy may be explained by differences in baseline characteristics of the enrolled patients. Importantly, cardiac event rates were low in the DIAD study and not significantly reduced by a MPI-based screening strategy. Nevertheless, in the small group of patients with abnormal MPI, a step-wise increase in event rates was observed with increasing MPI abnormality. Of note, hard event rates were $2 \%$ in patients with normal scans but were $12 \%$ in patients with at least moderately abnormal MPI scans. In contrast to the general asymptomatic diabetic population, these highrisk patients may benefit from screening, as also suggested by the bypass angioplasty revascularization investigation BARI 2 diabetes trial $[29,30]$. In this trial no survival benefit was shown in patients undergoing early coronary revascularization as compared to intensive medical treatment. However, among high-risk patients selected for coronary artery bypass grafting, prompt revascularization was associated with a lower rate of major cardiovascular events than medical therapy. Accordingly, these observations indicate that while routine screening for abnormal MPI may not be effective in asymptomatic patients with diabetes, selective screening strategies are warranted to identify the small but high-risk subgroup within this population. In this regard, our current study may provide valuable data for the design of such strategies. Assessment of vascular stiffness by means of PWV was shown to accurately identify patients with a high risk of severe MPI defects. Accordingly, further screening in patients with elevated PWV may be recommended. On the other hand, when using a slightly lower cut-off value, PWV was also shown to have a high negative predictive value, indicating that PWV can accurately rule out severe MPI defects. Therefore, further evaluation in patients with a negative PWV study may be safely omitted.

Due to its low cost and noninvasive nature, PWV may be a practical first-line tool to differentiate patients with a higher and lower likelihood of having abnormal MPI. A number of limitations must be acknowledged in the current study. Some of the observed MPI defects may be attributed to artifact attenuation. However, regional wall motion on gated SPECT images was analysed for optimal differentiation between true MPI defects and attenuation artifacts. Evidently, larger prospective studies are needed to demonstrate the effectiveness of this strategy in terms of cost and outcome.

\section{Conclusion}

Vascular stiffness as noninvasively assessed by PWV is related to severely abnormal myocardial perfusion in asymptomatic patients with diabetes. Accordingly, PWV could be a practical tool to identify patients at higher risk of $\mathrm{CAD}$ and who could benefit from further screening.

Funding J.J. Bax received grants from Biotronik, BMS Medical Imaging, Boston Scientific, Edwards Lifesciences, GE Healthcare, Medtronic and St. Jude Medical.

J.W. Jukema received research grants from, and was speaker at (CME-accredited) meetings sponsored by, Astellas, Astra-Zeneca, Biotronik, Boston Scientific, Bristol Myers Squibb, Cordis, Daiichi Sankyo, Ely Lilly, Medtronic, Merck-Schering-Plough, Pfizer, Orbus Neich, Novartis, Roche and Servier.

\section{Conflicts of interest None.}

Open Access This article is distributed under the terms of the Creative Commons Attribution Noncommercial License which permits any noncommercial use, distribution, and reproduction in any medium, provided the original author(s) and source are credited.

\section{References}

1. Wild S, Roglic G, Green A, Sicree R, King H. Global prevalence of diabetes: estimates for the year 2000 and projections for 2030 . Diabetes Care. 2004;27(5):1047-53.

2. American Diabetes Association. Consensus development conference on the diagnosis of coronary heart disease in people with diabetes: 10-11 February 1998, Miami, Florida. Diabetes Care 1998;21(9):1551-9.

3. Kang X, Berman DS, Lewin HC, Cohen I, Friedman JD, Germano $\mathrm{G}$, et al. Incremental prognostic value of myocardial perfusion single photon emission computed tomography in patients with diabetes mellitus. Am Heart J. 1999;138(6 Pt 1):1025-32.

4. Miller TD, Rajagopalan N, Hodge DO, Frye RL, Gibbons RJ. Yield of stress single-photon emission computed tomography in asymptomatic patients with diabetes. Am Heart J. 2004;147(5):890-6.

5. Young LH, Wackers FJ, Chyun DA, Davey JA, Barrett EJ, Taillefer $\mathrm{R}$, et al. Cardiac outcomes after screening for asymptomatic coronary artery disease in patients with type 2 diabetes: the DIAD study: a randomized controlled trial. JAMA. 2009;301(15):1547-55.

6. Lakatta EG, Levy D. Arterial and cardiac aging: major shareholders in cardiovascular disease enterprises: Part I: aging arteries: a "set up" for vascular disease. Circulation. 2003;107(1):139-46.

7. Laurent S, Cockcroft J, Van Bortel L, Boutouyrie P, Giannattasio C, Hayoz D, et al. Expert consensus document on arterial stiffness: methodological issues and clinical applications. Eur Heart J. 2006;27(21):2588-605.

8. Stehouwer CD, Henry RM, Ferreira I. Arterial stiffness in diabetes and the metabolic syndrome: a pathway to cardiovascular disease. Diabetologia. 2008;51(4):527-39.

9. Mattace-Raso FU, van der Cammen TJ, Hofman A, van Popele NM, Bos ML, Schalekamp MA, et al. Arterial stiffness and risk of coronary heart disease and stroke: the Rotterdam Study. Circulation. 2006;113(5):657-63.

10. Weber T, Auer J, O'Rourke MF, Kvas E, Lassnig E, Berent R, et al. Arterial stiffness, wave reflections, and the risk of coronary artery disease. Circulation. 2004;109(2):184-9.

11. Weber T, Auer J, O'Rourke MF, Kvas E, Lassnig E, Lamm G, et al. Increased arterial wave reflections predict severe cardiovascular 
events in patients undergoing percutaneous coronary interventions. Eur Heart J. 2005;26(24):2657-63.

12. Williams B, Lacy PS, Thom SM, Cruickshank K, Stanton A, Collier D, et al. Differential impact of blood pressure-lowering drugs on central aortic pressure and clinical outcomes: principal results of the Conduit Artery Function Evaluation (CAFE) study. Circulation. 2006;113(9):1213-25.

13. Willum-Hansen T, Staessen JA, Torp-Pedersen C, Rasmussen S, Thijs L, Ibsen $\mathrm{H}$, et al. Prognostic value of aortic pulse wave velocity as index of arterial stiffness in the general population. Circulation. 2006;113(5):664-70.

14. Rose G, McCartney P, Reid DD. Self-administration of a questionnaire on chest pain and intermittent claudication. $\mathrm{Br} \mathrm{J}$ Prev Soc Med. 1977;31(1):42-8.

15. Expert Committee on the Diagnosis and Classification of Diabetes Mellitus. Report of the expert committee on the diagnosis and classification of diabetes mellitus. Diabetes Care 2003;26 Suppl 1: S5-20.

16. Hansen CL, Goldstein RA, Akinboboye OO, Berman DS, Botvinick EH, Churchwell KB, et al. Myocardial perfusion and function: single photon emission computed tomography. J Nucl Cardiol. 2007;14(6):e39-60.

17. Petretta M, Acampa W, Evangelista L, Daniele S, Ferro A, Cuocolo A. Impact of inducible ischemia by stress SPECT in cardiac risk assessment in diabetic patients: rationale and design of a prospective, multicenter trial. J Nucl Cardiol. 2008;15 (1):100-4.

18. Wilkinson IB, Fuchs SA, Jansen IM, Spratt JC, Murray GD, Cockcroft JR, et al. Reproducibility of pulse wave velocity and augmentation index measured by pulse wave analysis. J Hypertens. 1998;16(12 Pt 2):2079-84.

19. Oliver JJ, Webb DJ. Noninvasive assessment of arterial stiffness and risk of atherosclerotic events. Arterioscler Thromb Vasc Biol. 2003;23(4):554-66.

20. Vlachopoulos C, Aznaouridis K, Stefanadis C. Prediction of cardiovascular events and all-cause mortality with arterial stiffness: a systematic review and meta-analysis. J Am Coll Cardiol. 2010;55(13):1318-27.

21. Cruickshank K, Riste L, Anderson SG, Wright JS, Dunn G, Gosling RG. Aortic pulse-wave velocity and its relationship to mortality in diabetes and glucose intolerance: an integrated index of vascular function? Circulation. 2002;106(16):2085-90.

22. Hatsuda S, Shoji T, Shinohara K, Kimoto E, Mori K, Fukumoto S, et al. Regional arterial stiffness associated with ischemic heart disease in type 2 diabetes mellitus. J Atheroscler Thromb. 2006;13 (2):114-21.

23. Fukui M, Kitagawa Y, Nakamura N, Mogami S, Ohnishi M, Hirata $\mathrm{C}$, et al. Augmentation of central arterial pressure as a marker of atherosclerosis in patients with type 2 diabetes. Diabetes Res Clin Pract. 2003;59(2):153-61.

24. Fantin F, Mattocks A, Bulpitt CJ, Banya W, Rajkumar C. Is augmentation index a good measure of vascular stiffness in the elderly? Age Ageing. 2007;36(1):43-8.

25. Hope SA, Antonis P, Adam D, Cameron JD, Meredith IT. Arterial pulse wave velocity but not augmentation index is associated with coronary artery disease extent and severity: implications for arterial transfer function applicability. J Hypertens. 2007;25 (10):2105-9.

26. Hope SA, Tay DB, Meredith IT, Cameron JD. Use of arterial transfer functions for the derivation of central aortic waveform characteristics in subjects with type 2 diabetes and cardiovascular disease. Diabetes Care. 2004;27(3):746-51.

27. Lacy PS, O'Brien DG, Stanley AG, Dewar MM, Swales PP, Williams B. Increased pulse wave velocity is not associated with elevated augmentation index in patients with diabetes. J Hypertens. 2004;22(10):1937-44.

28. Wackers FJ, Young LH, Inzucchi SE, Chyun DA, Davey JA, Barrett EJ, et al. Detection of silent myocardial ischemia in asymptomatic diabetic subjects: the DIAD study. Diabetes Care. 2004;27(8):1954-61.

29. Chaitman BR, Hardison RM, Adler D, Gebhart S, Grogan M, Ocampo S, et al. The Bypass Angioplasty Revascularization Investigation 2 Diabetes randomized trial of different treatment strategies in type 2 diabetes mellitus with stable ischemic heart disease: impact of treatment strategy on cardiac mortality and myocardial infarction. Circulation. 2009;120(25):2529-40.

30. Frye RL, August P, Brooks MM, Hardison RM, Kelsey SF, MacGregor JM, et al. A randomized trial of therapies for type 2 diabetes and coronary artery disease. N Engl J Med. 2009;360 (24):2503-15. 